\title{
Challenges in Combining Indigenous and Scientific Knowledge in the Arctic
}

Olga Lauter

École des Hautes Études en Sciences Sociales (EHESS)

\begin{abstract}
A "co-production of knowledge" transdisciplinary approach connects different systems of knowledge that are in collaboration with each other. The transdisciplinarity presupposes bringing natural, social sciences and Indigenous knowledge together. Education research in the Arctic plays a key role in bridging Western science and Indigenous knowledge systems, as it seeks for methods to integrate Indigenous knowledge systems into schools and postsecondary institutions to develop sustainability. Even though Indigenous knowledge systems are considered to be essential for sustainable development and environmental management (Athayde et al. 2017), connections between Indigenous peoples, scientists, politicians are not yet sufficiently elaborated (Raymond at al. 2010).

This paper examines several observations carried out during an ethnographic field work on urban Indigenous identity preservation and well-being in Anchorage, Alaska. The fieldwork in progress is being implemented in collaboration with the urban Yupik population. It throws light on challenges that might hinder the synthesis of Indigenous knowledge and Western science. For instance, one of the challenges under consideration has been that, while permissions to carry out participant observations were always easily obtained, allowing for building relationships and trust, the target population was, in most cases, reluctant when asked to be interviewed. While searching for the reasons of the unwillingness to participate in interviews, it became clear that the Alaska Native People do not trust researchers coming to work with them for a short period of time. Hence, long fieldworks should be implemented in order to have time to build trust. Another reason indicated is that the Alaska Native People learn through observing and listening to Elders, they do not like to consider themselves as experts. Thus, being interviewed is considered as showing expertise (Augusta Reimer, personal communication 2019). The observations examined in this paper will contribute to further understanding of differences in learning between Arctic Indigenous and Western knowledge systems.
\end{abstract}

Key Words: co-production of knowledge; sustainability; epistemological pluralism; Indigenous; Western knowledge

\section{INTRODUCTION}

Collaboration between Western scientists and traditional knowledge-bearers, Indigenous and sustainability sciences have a potential of offering one another techniques and methods for sustaining resilience in a rapidly changing social-ecological Arctic environment (Johnson et al., 2016). Even though Indigenous knowledge systems are considered to be essential for sustainable development and environmental management (Athayde et al. 2017), connections between Indigenous peoples, Western scientists, politicians are not yet sufficiently elaborated (Raymond at al. 2010).

Challenges in approach to knowledge of Indigenous people can be observed in diverse sustainabilityrelated scientific literature, starting with its diverse terminology: there is no unanimous definition of Indigenous knowledge: traditional (ecological) knowledge, local knowledge, Indigenous science, Native ways of knowing in parallel with Western science and scientific knowledge (Berkes 1993, Kawagley 1997, Bernhardt and Kawagley, 2005, Johnson et al. 2016, Lavrillier and Gabyshev 2017). For instance, while the word 'traditional' emphasizes a cultural continuity, it refers to the past, ignoring the adaptation of Indigenous communities to a constant change. 'Local' refers to the place-based type of knowledge but "it lacks specificity" (Mazzocchi 2006). Thus, the Indigenous knowledge systems 
remain difficult to define using a universal term. In this article, we will use the terms "traditional knowledge (systems) ”, “Indigenous science” and "Indigenous knowledge” as synonyms together with "Western science" and "Western knowledge systems" to put an emphasis on their equality.

Traditional and Western knowledge systems have important similarities: they both rely on observation, experimentation, pattern recognition, skepticism of second and third-hand sources, creativity and intuition. Some Indigenous people see the collaboration with Western scientists as a full of potential, necessary, complementary partnership to balance two different approaches on the condition for both of them to be considered as equal (Martinez and Kawagley in Merculieff and Roderick 2013). Others have distrust in distinct and historically dominant academic research traditions and methodologies. At the same time, some members of Western academic world either reject the idea of integration of Indigenous knowledge and Western science claiming that further integration effort may result in more power asymmetry and Indigenous knowledge marginalization or refuse to consider Western scientists and traditional knowledge-bearers as compatible and equal partners (Du Toit 2004, Johnson et al. 2016).

Together with similarities, values and approaches behind Western science and Indigenous knowledge systems (or Indigenous science) have considerable differences that may remain an obstacle to a successful collaboration of knowledge systems. However, creating and encouraging opportunities for a dialogue by working towards building trust between two partners are needed by both sides. In the first part of this article, we will focus on some observations carried out during our recent ethnographic fieldwork among the urban Yupik population that directed us to reflecting on the obstacles, challenges and ethical dilemmas faced while requesting to conduct our research among the Anchorage Yupik community. In the second part, we will examine differences and similarities between Western science and Indigenous knowledge systems. Finally, we will elaborate on Indigenous approach to learning and its role for education as a part of transdisciplinary approach and the co-production of knowledge.

\section{INDIGENOUS KNOWLEDGE AND EPISTEMOLOGICAL PLURALISM}

Bridging Indigenous and scientific knowledge has its specific challenges to concentrate on. Even though Western sciences may share more common values and approaches, difficulties remain within Western interdisciplinary research itself, despite efforts to overcome barriers to communication between different knowledge systems and progress associated with these efforts. Challenges in our recent two-year ethnographic fieldwork in Anchorage, Alaska motivated us for search of new concepts that could help non-Indigenous researchers to decolonize their research methodology in order to inspire a better co-production of different knowledge systems. The concept of epistemological pluralism is an approach for collaboration and innovative research aiming at achieving transdisciplinarity by avoiding disciplinary-dominated research (Miller et al. 2008). This concept seems to be an indispensable step for successful collaboration of ways of knowing. Transdisciplinary research presupposes the transcendence of disciplines that relate to each other while constituting one whole system without rigid borders (Athayde et al. 2017). It is possible to implement by means of recognizing that a particular way of knowing is not sufficient to understand the world complexity, thus respect and regular negotiation of research's values and aims need to be conducted on a regular basis in order to prevent researchers' tendency to privilege one epistemology over another (Miller et al. 2008). Very important in this regard, as far as the integration process is concerned, is flexibility allowing for changes in perceptions during a project to be discussed between bearers of different knowledge systems. This approach needs to be applied while conducting research in collaboration of several knowledge systems. It can be used in 
implementing collaboration between Western science disciplines but it should also be applicable to building bridges and to integration of Indigenous and Western sciences. Despite the fact that some literature indicates important risks of knowledge integration (Berkes et al. 2006, Johnson et al. 2016), Shuttenberg and Guth argue (2015) that, for a knowledge co-production to be a success, the appropriate necessary level of integration needs to be defined and achieved. Thus integration should allow for such a transformation in thinking that main stakeholders are able to accept values and ideas of other disciplines and cultures to search for and to find new, creative solutions to complex problems. Our ethnographic fieldwork experience served as a platform for discovering and reflecting on similarities, differences and challenges of collaboration between Western or non-Indigenous and Indigenous sciences that resulted in readjusting our research methods and ethical stands.

\section{DISCUSSION: CHALLENGES AND APPROACHES IN BRIDGING INDIGENOUS KNOWLEDGE AND WESTERN SCIENCE}

In this section, we reflect on challenges of collaboration between Western scientists and Indigenous people of the Arctic and on approaches to overcome the challenges that emerged as a consequence of ethnographic fieldwork that has been carried out in Anchorage, Alaska between 2018 and 2020. One of the challenges encountered during this recent fieldwork focused on negotiation of cultural and religious identity of the urban Yupik population was a contradiction between, on the one hand, receiving permissions to carry out research easily, thus, enabling to observe various activities, to participate in different events of the community and to build relationships. Hence, the urban Yupik community has been open to welcome us as a learner. On the other hand, the target population, in general, has been reluctant to participate in semi-structured interviews being cautious about signing a consent form followed by an approximately one-hour discussion. While searching for the reasons of the unwillingness to be interviewed, several issues came to light.

\section{a) Question of trust}

To start with, it became clear that the Alaska Indigenous people mistrust non-Indigenous researchers. It is common knowledge that Western researchers have become a source of distrust for Indigenous people due to previous and still existing research methods and practices. Stories about research and researchers are associated in Indigenous communities with stories about colonization and injustice (Smith 1999). Researchers from various disciplines approach and conduct surveys with Indigenous people on a regular basis. Besides, local and foreign journalists, writers, photographers and tourists attempt to reach Indigenous people for the purpose of writing articles, reports, fiction, of conducting photo exhibitions and filming. Thus, sometimes Indigenous people do not recognize a difference between a researcher asking for a permission to carry out a long-term project and a journalist or a writer aiming at exclusively conducting a series of interviews in order to write a newspaper article or a fiction piece (Anonymous, personal communication, 2019). Hence they become overwhelmed with various outsiders willing to gather data in the communities. Indigenous communities consider themselves to

“ have been researched to death” (Kawagley 1997). What are the other reasons, why some Indigenous communities still remain so skeptical about Western science?

As it was in the past, scholars from around the world continue to travel to rural and urban northern Indigenous communities, for instance, in order to complete their field work for a PhD degree or a shorter-term scientific project. Some foreign scholars come to the field for a short and limited amount of time, often with little funding available. Numerous resources and ethical guidelines (for instance 
National Science Foundation guidelines) exist for conducting research with Northern communities. Indigenous communities establish their own ethics guideline and review boards in addition to United States and foreign institutional review boards. These measures aim to provide ethical training to scholars and to contribute to protection of Indigenous communities from unethical methods and practices. However, first, ethical boards and training do not exist in all European institutions. Thus, for instance, some foreign scholars, who have little funding to travel for research in Northern communities but who need to collect data for writing and defending their doctoral thesis, choose to conduct a shortterm research project. Hence they face the challenge of completing their field project with no or little ethical training and limited or no funding to share with these communities for the collaboration in research projects. Such cases lead Indigenous communities to further distrust of researchers that "take without giving back" (Cochran et al. 2008). Second, although ethical trainings and requirements, while being a means of protection against unethical practices in Indigenous communities do not seem to resolve all the potential ethical dilemmas, partially, due to their cultural insensitivity. For instance, according to Smith (1999), a consent form, that is required for a researcher to present to a potential interviewee to be read and signed, thus, a requirement to give consent to be interviewed, may be considered as a confirmation of "trust as a static decision" refusing any possibility of its requestioning or renegotiation. There were some instances during our fieldwork, where we were allowed to learn from the Indigenous community members by asking them questions in an informal setting, but when it came to trying to have the consent form read and signed, we were indirectly refused. Furthermore, we claim that often we learned more by informal interviews than by conducting formal interviews with the Indigenous community members. But, if no formal consent for using the data is given, it can serve an anthropologist as a source of understanding cultural aspects but, at the same time, they have no legal and no ethical right to publish by adding the acquired data. Thus, despite the existing institutional and local review boards installed, a lot of ethical dilemmas remain unsolved in collaboration of Western science and Indigenous knowledge-bearers. It may lead to hindering the quality of collected data, as Indigenous knowledge-bearers, by accepting the procedure of giving the official consent to a formal interview, may become passive and uninterested interviewees "playing the game of non-Indigenous research" (Smith 2008). Thus, more discussion between Western and Indigenous scientists and institutional boards may be needed to find a format of collaboration that is more culturally sensitive.

\section{b) Knowledge sharing}

Furthermore, some Indigenous communities consider that the main researchers' goal to gather data is ambitious and carrier-oriented (Kawagley 1997). Researchers still come to an Indigenous community with ready hypotheses and questions to discuss, thus, collecting data to earn a degree or to accomplish a project for specific career purposes. If they consider traditional knowledge collection exclusively as a personal gain, not only this kind of approach does not contain any benefit to Indigenous communities, it also contradicts community values, according to which knowledge is accumulated to be transmitted not only for individual but also for community future use by future generations. According to Smith (1999), researchers gather data that are already known, they make suggestions that would not work, then they return to their country to write up their fieldwork report or to write a dissertation without sharing study results with the community. Although Indigenous communities maintain friendly and respectful relationships with non-Indigenous scholars, researchers' work may be perceived by the communities as worthless (Smith 1999). Hence, Indigenous people may have no motivation to collaborate with researchers, as they may not expect the results to be shared with them and they may not believe that the results may be beneficial for them. 


\section{c) Fear of misinterpretation}

Additionally, some more important challenges arise that should be reflected on before and during a project implementation: the possibility of misinterpretation, the necessity of sharing gathered information, and confidentiality. Fear of data misinterpretation is one of the sources of distrust of researchers by Indigenous communities. It needs to be recognized and addressed by academia (Athayde et al. 2017). Western researchers interpret traditional Indigenous knowledge from a Western perspective. A scientist shapes their research through the prism of their culture. Thus, according to the Indigenous perspective, Western science distorts traditional knowledge shared leading to data misinterpretation (Cochran et al. 2008). Indeed, some of our Indigenous partners agreed to collaborate with a remark that "no information gathered should be misinterpreted" (Anonymous, personal communication, 2019). Thus, to avoid data misinterpretation, a possibility of sharing them before publishing could be suggested to the Indigenous collaborators at the early stage of a project discussion. Indigenous partners could be given a choice of means by which the data can be shared. For instance, it can either be an oral presentation in front of the whole community or data can be shared in the written form, so that they are available for individual or collective reading by interested community members. By explicitly explaining an obligation to share the results gathered, the risk of misinterpretation is being diminished, which may seem to allow for better exchange outcomes and for building of trust. Some of our Indigenous collaborators agreed on interviews to be recorded under the condition of being able to read through the data before their publication. Thus, such an approach permits simultaneously to build a better trust while obtaining more accurate data by their verification with Indigenous partners. However, one of the challenges for sharing the data with Indigenous collaborators consists in the fact that, for instance, in some foreign institutions, for instance, in France, doctoral dissertations are required to be written in the native language of a respective country. Thus, extra funding and time need to be anticipated for translations.

\section{d) Confidentiality issues}

Challenges of confidentiality are closely connected to the question of research results sharing. How to maintain confidentiality, if the research results are shared for a review to members of the same possibly small- community before a publication, if they often can identify each other, even if the anonymity is respected (Ellis 1995)? A researcher needs to filter the data according to the ethical perspectives in reflecting in advance on what kind of material and to what extent research result publications might potentially harm the community members. Research outcomes to publish need to be chosen in a careful and ethical manner, even though not all the community members require outcome review before a publication. For instance, we had a collaborator we became friends with in the course of our recent fieldwork. Some information was given informally but, as it came to an interview request, the collaborator hesitated to be formally interviewed. They agreed for an interview under the condition to remain anonymous. However, they were worried about their perspective to be shared and disclosed in a paper form, as the community members and other potential audience might recognize them by the manner the perspective would be shared. Hence in order to build and to maintain trust, researchers need to consider Indigenous communities, they collaborate with, as potential readers of their publications, by filtering the data gathered in an ethical manner that prevents collaborators from an exposure to any potential harm or risk. 


\section{e) Relationship-building and temporality}

Short-term projects are one of the further factors contributing to the distrust of researchers by Indigenous communities. Relationship-building is a long, time-consuming process. As mentioned earlier, some researchers coming to complete their projects in Indigenous communities often do not have enough funding for a long-term stay. This challenge concerns, in particular, early career researchers pursuing doctorate degrees and coming from abroad. However, time is a crucial factor for building relationships between Western and Indigenous scientists (Brook and Stéphane M. McLachlan 2005). For our target population, the urban Yupik Indigenous people as well as for other Indigenous people in Alaska and in the Arctic, building of long-term relationships is indispensable for developing trust. As Haig-Brown (2003) puts it, "when [Western researchers] allow themselves to recognize our limitations and to take the time to really listen to people who are not just like us... they can learn to listen differently to the words of study participants”. We agree that the approach is beneficial for both sides, if Western researchers consider themselves as learners, on the one hand, from the very first stage of their research in such a manner, as they introduce themselves to an Indigenous community to conduct a study, and, on the other hand, the Indigenous community's teaching role is clearly announced. In defining the roles from the very beginning in such a manner, that both sides have in a research project, allows for a teaching and learning relationship development that presupposes having a trustful relationship. Indigenous teachers and learners often have close and long-lasting relationships through common ancestors, land and waters (Merculieff and Roderick 2013). Researchers, coming to collaborate from abroad, may not be worth investment into relationships from the Indigenous perspective, as they usually have a short-term objective to complete and then they go back home (Augusta Reimer, personal communication, 2019). Without trust, a long-term and nurturing dialogue between a researcher and an Indigenous community, bridging Western science and traditional knowledge is difficult to implement. The possible outcome for scientists for a short-term project could be incomplete, superficial or even incorrect data, hindering the quality of the fieldwork conducted. Hence, the more researchers invest into spending time for building long-term relationships with Indigenous communities, the better the chances are for the academia to open and to develop a dialogue with traditional knowledge-bearers. There is a possibility to initiate and develop long-lasting relationships between researchers and Indigenous communities, if funding agencies understand the reasoning and importance behind supporting community participatory research studies over an extended period of time (Chilisa 2012). Thus, researchers need to include solid argumentation for increasing and justifying the costs related to lengthening project in order to build trustful relationships with Indigenous communities into their budgets to examine by funding agencies.

\section{f) Concept of expertise}

One of the differences in Indigenous traditional knowledge and Western science approaches that might hinder a successful bridging of both is the relationship to the concept of expertise. For instance, Inupiat and Yupik Indigenous traditional knowledge bearers - Elders - are considered to be experts by right by their community but, according to one of the Indigenous values - the importance of humility - they will never consider themselves as such (Augusta Reimer, personal communication, 2019, Beans 1997). Being an Elder is a status that an Indigenous community grant for knowledge and wisdom, they need a lifetime to acquire and to share it (Merculieff and Roderick 2013, Hild 1994). In contrast, in Western science, earning a degree and acquiring a higher position in the academia world presupposes a transition to a certain level of expertise. Much longer time is needed to become an Elder than to earn a 
degree. Thus, a researcher's goal to complete a short-term or long-term project in order to become an expert may be skeptically seen by knowledge-bearers of an Indigenous community (Augusta Reimer, personal communication, 2019). This limitation of Western science may become an obstacle to the openness of scientific inquiry by focusing on the questions, funding is secured for by a powerful group of experts within academic structures (Johnson et al. 2016).

One of the ways we chose to invite Elders and other members of Indigenous communities to a dialogue about Western science and Indigenous knowledge expertise with the purpose of trust-building was to learn the values of the respectful target population and to collaborate with it while respecting and practicing the values in the community. On the one hand, this approach requires decolonization and contextualizing of Western science thinking in new ways (Ibid.). On the other hand, the effort risks to remain insufficiently efficient, if only one side tries to reach for understanding the other. However, taking into consideration the fact that Western scientists are a part of institutions historically associated with colonization and still being situated in the position of power, it is researchers' responsibility to make the first steps towards Indigenous communities of different knowledge systems by aiming at an attempt to understand the other, even though both sides are in need of a collaboration facing the problem of climate change.

In order to achieve this goal, we began with analyzing strengths and limitations of different knowledge systems, while searching for similarities to find the common ground for connection and for boosting a further dialogue. In our opinion, for the Western science, the past and current authoritative and dominant nature of its research still needs to be openly recognized and awareness about the past practices and colonized nature of research should be maintained during the whole process of a collaboration. Employing the worldviews and values of a target population requires time investment, thus, academic institutions and funding agencies need to support researchers in the effort to spend time on developing relationships and learning the appropriate behaviors with the help of community members before determining research methodologies. For example, during our recent fieldwork, we have intentionally used the word 'learning' while explaining the details and goals of our project to several members of the community to work with. Such a manner to present the project turned out to facilitate its acceptance by some Indigenous collaborators. Thus, one of the ways to focus on building long-term relationships with an Indigenous community could be communicating a researcher's intention and motivation to learn on a long-term basis and not exclusively for one project. Another important point, according to what we have drawn as a conclusion from our recent and current fieldwork experience, is that non-Indigenous researchers may constantly need to reflect on the idea of the colonization as a culture that is still shared by both "the colonized" and "those who have colonized" (Smith 1999). Even though we were ready to readjust our research focus, questions and hypothesis, as a result of a dialogue we hoped to lead with the Indigenous community, we must admit to have had little understanding of how tense the relationship between different knowledge systems is partially due to their common "struggle for decolonization” (Ibid.).

\section{DIFFERENCES AND SIMILARITIES IN INDIGENOUS AND WESTERN KNOWLEDGE SYSTEMS}

In this section, we would like to elaborate on main differences between Western and Indigenous sciences, but we would also like to examine and to draw attention to similarities between two of them. This comparative analysis can be characterized rather as indicative than definitive, as Western and Indigenous knowledge systems differ (Barnhardt and Kawagley 2005). Generally, one of the most significant differences between both sciences or knowledge systems is their goals to achieve. Western 
science seeks to understand to predict the nature in order to control it by creating technologies, while Indigenous science seeks to understand the natural world in order to adapt to it (Merculieff and Roderick 2013). As for the methods applied, traditional knowledge combines collective knowledge, that is specific to a place, with individual long-term observations, in other words, the knowledge is high-contextual and local. Furthermore, Indigenous science is interrelated with spirituality and wisdom (Johnson et al. 2016, Kawagley 1997), while Western knowledge seeks for objectivity and detachment. Also, Western science is fragmentary by nature, it is mostly focused on examining parts of the whole in order to reconstruct the global picture, while Indigenous science is holistic and it is focused on relationships and connectedness of all the parts of the whole (Ibid.). In addition, traditional knowledge is passed on to future generations by Elders by means of the oral tradition, while Western knowledge is about documenting collected data, thus, literal. Western science is considered by some researchers as theoretical in contrast with Indigenous science being practical (Barnhardt and Kawagley 2005), although other researchers recognize traditional knowledge as theoretical and analytical (Lavriller and Gabyshev 2017).

Despite significant differences, there are similar aspects that Western and Indigenous scientists have in common (Martinez in Merculieff and Roderick 2013). They observe and experiment, they both often use specific language terms (Lavrillier and Gabyshev 2017). They recognize patterns, question and test their hypothesis. They use creativity and intuition. Both Western and Indigenous scientists doubt and test the research results coming from external sources. Understanding of the world is implemented by learning. Minimizing differences and focusing on similarities allows for knowledge co-production (Schuttenberg and Guth 2017). We believe that, despite significant differences and in order to reach an open dialogue, better chances exist to bridge both sides by paying special attention to commonalities while recognizing, indicating, taking into consideration and discussing differences. Also, as Barnhardt and Kawagley (2005) note, Indigenous knowledge systems' diversity, development and adaptation to a constant change, on the one hand, and the development of Western sciences that enables their better understanding of and growing interest in Indigenous sciences, on the other hand, lead to an increasing recognition of the complementarity that exists between two knowledge systems that were considered as very different before.

\section{INDIGENOUS APPROACH TO THE CONCEPT OF LEARNING AND ITS ROLE FOR EDUCATION IN A RAPIDLY CHANGING ARCTIC}

\section{a) Indigenous concept of learning}

In this section, we will elaborate on Indigenous approach to the concept of learning and on its role for public and institutional education. Mutual learning is a fundamental aspect of collaborative research as it enables to understand others' epistemological positions and as it helps to analyze how they impact knowledge integration (Athayde et al. 2017, Raymond et al. 2010). Also, since constant learning is one of the values of the Yupik and other Alaskan Indigenous people, it seems important to focus on understanding specificities of the Indigenous concept of learning in an attempt to bridge the gap between different knowledge systems and to look for methods to reduce mistrust between Western scientists and traditional knowledge-bearers. While the approaches of traditional and Western science knowledge systems to learning are different, their better mutual understanding by both sides is likely to contribute to a better dialogue. Thus, there is a possibility for "improving the transdisciplinarity reach of sustainability science through engaging Indigenous sciences” (Johnson et al. 2016). 
Learning in the Indigenous cultures is often considered as a life-time process. People learn at an individual rate, and the learning process is not imposed upon anyone, but it is personal and it rather depends on self-motivation. A person may return to the same teaching again and again during their lifetime to learn new details according to an individual experience progressively gained (Wright 2010). Western science researchers are often constrained by time frames and limitations of research projects they need to accomplish, that is why, as difficult as the task is, long-lasting research opportunities enabling to return and to work with the same communities need to be foreseen.

Non-verbal communication is a very important part of teaching and learning connected to active participation and observation in Indigenous communities of Alaska as well as to the question of nonauthoritative knowledge (Pingayak 2003). According to Merculieff (2013), instead of words that limit intelligence or ability to learn, Indigenous teachers' main task is to provide learning opportunities. Direct questioning and answers are discouraged, little direct instruction is being used, few detailed explanations are provided. The more specific the question is, the narrower the response will follow. Instead of giving a direct and definitive answer, a story may be told as an illustration. And it is up to a learner which lesson to draw. "When I talk here with some of the professors or a patriarchal authority figure, I get disempowered. The Elders don't do that. They practice a system of self-empowerment that lets me figure it out on my own" (Merculieff in Merculieff and Roderick 2013). Besides, learners are expected to observe, to listen respectfully, "without agenda”, not to interrupt a teacher. Listening is valued both in terms of traditional values of respect and too much conversation and discussion is considered as an obstacle to observing and experiencing. Also, non-verbal learning contributes to visual acuity and attention to detail that is crucial to daily observations of environment and the weather (Ibid.). Thus, Western partners need to take into consideration the importance of non-verbal communication and, consequently, a possibility of resentment being created by asking direct questions and awaiting direct answers. They need to rethink their interviewing methods, as the unstructured or non-standardized or semi-structured interviews might be easier to implement, as structured and standardized interviews (Chilisa 2012).

As for traditional Yupik learning, in the past, the Yupik Indigenous people learned by active participation in the field activity and observation. (Ilutsik 1999). The specificity of our fieldwork has consisted in the fact that, on the one hand, we did not live within the community researched, but we were rather in a close proximity to it on a regular basis. On the other hand, we maintained contact by participating in weekly events and activities of the urban Yupik people during a relatively long period of time of approximately two years. Thus, we experienced challenges of the community acceptance similar to those researchers who come to the Arctic communities for short-term projects and who have difficulties in building trust. At the same time, we had a possibility to engage in some of the community activities, to experience, to reflect on and to re-ask questions. Establishing relationships and learning about the community in our case has been a relatively long process. We learned that regular repetition of the same activities with the purpose of mastering and rethinking them is although sometimes a tedious but an important task in the process of the Indigenous way of teaching and learning. Hence, we participated in some weekly, monthly and annual events and their repetition allowed us to come up with new questions and to remodel and readjust our methodology and ethnographic problems. Researchers' active participation in the community activities combined with observations seem to be no less significant for a project success as interviews (Chilisa 2012). Although researchers are very often constraint in time and funding, the significance of investing time in community activities for the sake of the mutual learning development should be reflected on in advance and used in argumentation for grant proposals. 


\section{b) Education}

As for the relation of Indigenous approach to learning to public and institutional education, we would like to start first by discussing today's role of education in the Arctic and its place in interdisciplinary research. On the one hand, public school and institutional education and learning have significant potential in contributing to sustainability in the Arctic, as it is an indispensable component for its promotion (Lemons 1992). The public school and institutional systems can be detrimental to northern communities or it can offer students a significant potential of becoming resilient to changing environments (Cost 2015). Inclusion of epistemological pluralism, or, in other words, of diverse ways of knowing, and, in particular, of Indigenous knowledge systems into the curricula is indispensable for resilience and well-being of rural communities. Without the application of traditional knowledge and culture and place-based schooling into rural educational institutions, the value of education may be lost on them (Ibid.). Also, urban public schools and universities are in need of doing the same as a part of decolonizing and sustainability efforts. Indigenous ways of knowing with their focus on living in balance with the nature and their equal inclusion together with non-Indigenous knowledge systems may contribute to students' ability to better solving of complex social-ecological environmental problems in the future. On the other hand, education has an important role to play in the interdisciplinary approach to sustainability, as it can contribute to a further dialogue on knowledge co-production based, for instance, on the experience from attempting to combine knowledge systems through rural programs and initiatives (Bernhardt and Kawagley 2005). As for the previous observations on Indigenous approach to learning, anthropological fieldwork and project experiences of non-Indigenous researchers may contribute to the implementation methodologies on how non-Indigenous school personnel could approach to combining different ways of knowing in class. In its turn, it could contribute to decreasing high rural school personnel turnover (Cost 2015).

\section{CONCLUSION}

In spite of some challenges and ethical dilemmas in our ethnographic fieldwork experience demonstrated in a dialogue with the current literature, the article identifies both differences and similarities of Indigenous and non-Indigenous knowledge systems, taking into account that the comparisons are rather indicative than definitive. Focusing on the differences allows for better understanding of potential challenges of knowledge co-production. Similarities may be considered as a common ground to start building up a dialogue between different knowledge systems. This dialogue is enabled and based on the concept of epistemological pluralism that equally accepts different knowledge systems and that strives for innovation and collaboration in research to bridge the gaps between Indigenous and non-Indigenous knowledge in order to achieve a better knowledge integration and coproduction. A successful knowledge collaboration is impossible without decolonization and mutual learning of both Indigenous and Western sciences that may become complementary in tackling complex sustainability issues in the Arctic. However, we suggest that, taking into consideration the fact that Western scientific institutions have been associated with colonization, it is non-Indigenous researchers' responsibility to be the first to analyze and to accept Indigenous knowledge systems as equal. We came to implement our fieldwork among the urban Yupik population with very limited knowledge of current research methods that could facilitate collaboration with Indigenous communities but the challenges encountered enabled us to reflect on and to identify the issues of trust, of confidentiality, of fear of misinterpretation, of long-term relationship-building necessity, of the 
relationship to expertise as well as of Indigenous approach to learning. Their analysis allowed for a better collaboration with the urban Yupik knowledge-bearers and it may serve for a better knowledge integration in future projects. We agree with Johnson et al. (2016) that already existing and emerging methods to bring together different knowledge systems are not exhaustive and they may not be applicable to any given situation and disciplines. However, for instance, they could serve in knowledge integration while training of northern community school and university personnel in including Indigenous way of knowing into the curricula.

\section{References}

Athayde S., Silva-Lugo J., Schmink M., Kaiabi A., Heckenberger M. (2017). Reconnecting art and science for sustainability: learning from indigenous knowledge through participatory action-research in the Amazon. Ecology and Society. 22(2):36.

Barnhardt R., Kawagley A.O. (2005). Indigenous knowledge systems and Alaska Native ways of knowing. Anthropology and education Quarterly. 36(1):8-23.

Beans W. (1997). Learning put into cultural perspective. Sharing Our Pathways. 2(2).

Brook R.K., McLachlan S.M. (2005). On using expert-based science to "test" local ecological knowledge. Ecology and Society. 10(2):r3.

Chilisa B. (2012) Indigenous Research Methodologies. University of Botswana, SAGE, Los Angeles

Cochran A.L., Marshall C.A., Garcia-Downing C., Kendall E., Cook D., McCubbin L., Gover R.M.S. (2008). Indigenous ways of knowing. Implicatons for participatory research and community. American Journal of Public Health. 98(1): 22-27.

Cost D.S. (2015) The role of public education in governance for resilience in a rapidly changing Arctic. Ecology and Society 20(3): 29.

Haig-Brown C. (2003) Creating spaces: Testimonio, impossible knowledge, and academe. International Journal of Qualitative Studies in Education. 16(3): 415-433.

Ilutsik E. (1999) Traditional Yup’ik knowledge - lessons for all of us. Sharing Our Pathways. 4(4)

Johnson J.T., Howitt R., Cajete G., Berkes F., Louis R.P., Kliskey A. (2016). Weaving Indigenous and sustainability sciences to diversify our methods. Sustainability Science. 11: 1-11

Kawagley A.O. (1997) Active Reality Research. Sharing Our Pathways. 2(3-5)

Lavrillier A., Gabyshev S. (2017) An Arctic Indigenous knowledge system of landscape, climate, and human Interactions. Evenki Reindeer Herders and Hunters. Kulturstiftung Sibirien gGmbH, Fürstenberg/Haven

Merculieff I., Roderick L. (2013). Stop talking. Indigenous ways of teaching and learning and difficult dialogues in higher education. University of Alaska Anchorage 
Miller T.R., Baird T.D., Littlefield G., Kofinas G., Chaplin III F.S., and Redman C.L. (2008). Epistemological pluralism: reorganizing interdisciplinary research. Ecology and Society. 13(2):46.

Pingayak J.F. (2003). Reviving our cultures and languages: a Cup’ik perspective. Sharing Our Pathways. 8(1)

Raymond C.M., Fazey I., Reed M., Stringer L., Robinson G., Evely A.C. (2010). Integrating local and scientific knowledge for environmental management. Journal of Environmental Management. 91(8): 1766-1777

Schuttenberg H.Z., Guth H.K. (2015) Seeking our shared wisdom: a framework for understanding knowledge coproduction and coproductive capacities. Ecology and Society. 20(1): 15.

Smith L.T. (1999) Decolonizing methodologies: research and indigenous peoples. University of Otago Press and Zed Books, Dunedinand London

Wright M. (2010) Aspects of silence: when do traditions begin? In Barnhardt R, Kawagley AO. Alaska Native Education. Alaska Native Knowledge Network, Center for Cross-Cultural Sudies, University of Alaska Fairbanks 